\title{
Analysis of the EDIPO Temperature Margin During Current Ramp-Up
}

\author{
Claudio Marinucci, Marco Calvi, Luca Bottura, Francesca Cau, and Alfredo Portone
}

\begin{abstract}
The European dipole (EDIPO), currently under construction, will provide background magnetic fields of up to 12.5 $\mathrm{T}$ for tests of ITER high-current superconducting cables. The EDIPO winding consists of $7 \times 2$ double layers of $\mathrm{Nb}_{3} \mathrm{Sn}$ cable-in-conduit conductors with forced flow cooling of supercritical helium. The performance limits of EDIPO during current ramp-up are analyzed analysed with the CryoSoft suite of codes, recently integrated into a customizable and flexible environment for the analysis of thermal hydraulic and electrical transients in superconducting magnetic systems. The simultaneous analysis of the cryogenic system and all 14 double layers shows that under all charging conditions the EDIPO temperature margin remains sufficiently high.
\end{abstract}

Index Terms-CICC, ITER, modeling, superconducting magnets.

\section{INTRODUCTION}

$\mathbf{T}$ HE superconducting high-field European dipole (EDIPO), built in close collaboration of EURATOM, European associations and industries, will be integrated in a new test facility at CRPP next to the existing SULTAN facility. The EDIPO facility is expected to qualify the production of ITER full-size conductors and, more generally, to test superconducting conductor samples carrying currents of up to 100 $\mathrm{kA}$ in magnetic fields of up to $12.5 \mathrm{~T}$ [1]. The dipole will be delivered to CRPP at the end of 2010 [2].

The DC winding will store $\approx 16 \mathrm{MJ}$ of magnetic energy at the design current $I=17 \mathrm{kA}$. Before starting operation the magnet will be energized to full current, possibly at the fastest current ramp rate allowed by the dedicated power converter $(d I / d t \approx 50 \mathrm{~A} / \mathrm{s})$. During this process heat due to AC losses, which are proportional to $d I / d t$, is generated in the winding. The primary goal of this thermal hydraulic $(\mathrm{TH})$ analysis is to assess the EDIPO performance limits during current ramp-up through the temperature margin $T_{M}=T_{C S}-T$ in the complete winding, where $T_{C S}$ is the current sharing temperature and $T$ the conductor temperature.

\section{EDIPO DC WINDING}

The EDIPO DC winding consists of a pair of identical saddle-shape superconducting coils, i.e., upper and lower sub-

\footnotetext{
Manuscript received October 19, 2009. First published March 18, 2010; current version published May 28, 2010.

C. Marinucci, M. Calvi, and F. Cau are with the EPFL-CRPP, Fusion Technology, 5232 Villigen-PSI 5232, Switzerland (e-mail: Claudio.Marinucci@psi. ch).

L. Bottura is with CERN, AT-MSC, 1211 Geneva, Switzerland.

A. Portone is with Fusion for Energy, 08019 Barcelona, Spain.

Color versions of one or more of the figures in this paper are available online at http://ieeexplore.iee.org.

Digital Object Identifier 10.1109/TASC.2010.2040380
}

coil, which are layer wound using cable-in-conduit conductors (CICC) of two different cross sections, i.e., rectangular High Field (HF) and square Low Field (LF) conductors (Fig. 1). All conductors contain the same type of $\mathrm{Nb}_{3} \mathrm{Sn}$ strands. The non-copper critical current density $J_{c N C}=2000 \mathrm{~A} / \mathrm{mm}^{2}$ (@12 T, $4.2 \mathrm{~K}$ and longitudinal strain $\epsilon=-0.25 \%$ ) and the copper/non-copper ratio $r=1$ are the same in all conductors. The cable is jacketed inside a steel conduit. Each subcoil, made of 7 double layer (DL1-DL7 in the upper subcoil and DL8-DL14 in the lower subcoil), is cooled in parallel by a forced flow of supercritical helium.

\section{Simulation Model AND TOOLS}

\section{A. Model}

A model which includes all details of the EDIPO system would be too complex and expensive in computer time. Therefore, our model includes a number of simplifying assumptions as a compromise between reliability and cost efficiency. Some assumptions are slightly pessimistic, e.g., current and magnetic field are larger than the nominal values by a few $\%$, no jacket and insulation are included, etc. and some are slightly optimistic, e.g., the external radiation heat into the magnet is neglected since it is very low due to the cold shielding, the joint void fraction is larger than the nominal one, etc. The sensitivity of the results to variation of the reference set of physical and modeling input data is assessed parametrically.

1) DC Winding and Cryogenic System: All 14 double layers of the DC winding are modeled in 1-D along the conductor length (coordinate $X$ ). The CICC cable cross section model consists of $\mathrm{Nb}_{3} \mathrm{Sn}$, copper and helium whereas steel jacket and insulation are not included because of their negligible effect. $T_{C 0 M}=18 \mathrm{~K}, B_{C 20 M}=28 \mathrm{~T}, C_{0}=31.68910^{9} \mathrm{TA} / \mathrm{m}^{2}$ [4], filament diameter $d_{f}=80 \mu \mathrm{m}, \epsilon=-0.65 \%$ are the $\mathrm{Nb}_{3} \mathrm{Sn}$ data included in the model. Strand parameters of the power fit to the voltage-current curve for the critical current transition are: exponent $n=10$ and electrical field $E_{0}=$ $100 \mu \mathrm{V} / \mathrm{m}$. The copper residual resistivity ratio is $R R R=100$. The Dittus-Bolter correlation is used for the heat transfer coefficient model. Perfect thermal insulation is assumed between turns of the same and of adjacent double layers.

The cryogenic system is approximated by a simplified closed loop system which includes a pump (10 g/s), two heat exchangers to keep the helium temperature constant at the subcoil inlets $(4.5 \mathrm{~K})$ and a controller to keep the helium pressure constant at the pump inlet (4.0 bar). The pressure drop is $3 \mathrm{bar}$ in each subcoil (6 bar in the DC winding) [3]. A similar model was validated in the analysis of the ITER TF Model Coil [5]. A selection of coil parameters relevant for this study is given in Table I, and a scheme of the model is shown in Fig. 2. 


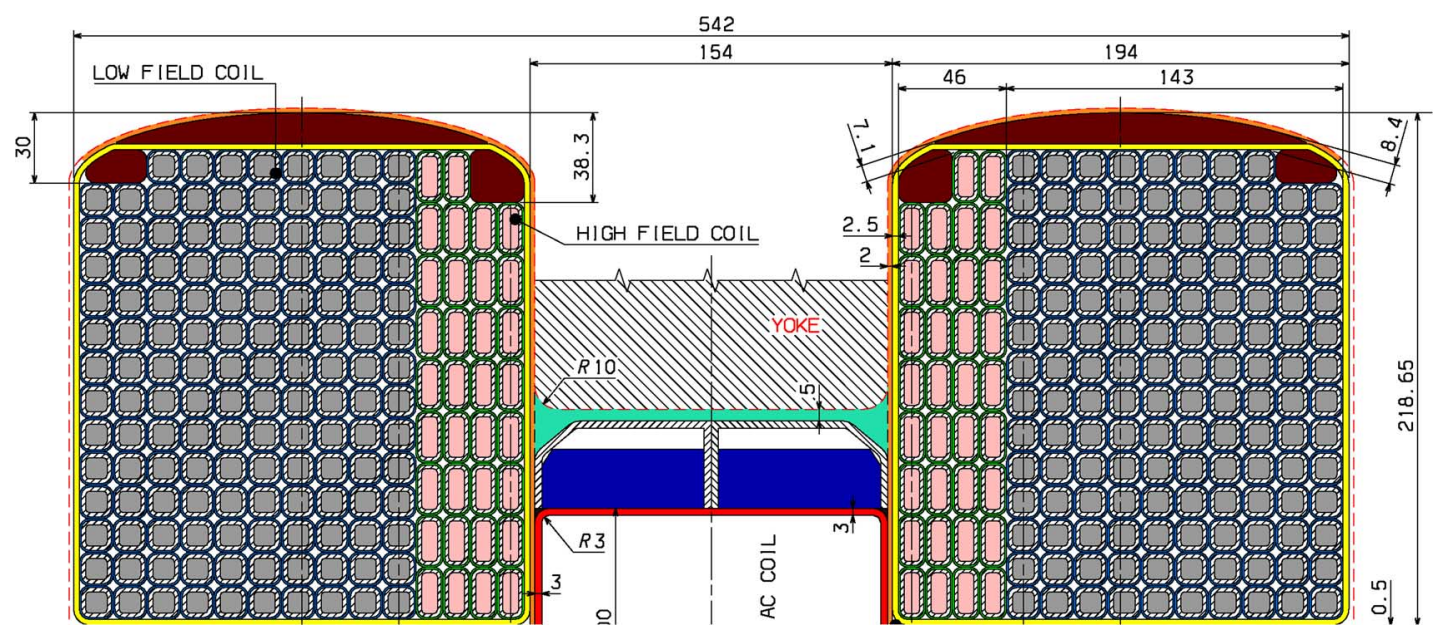

Fig. 1. Cross section of the EDIPO DC winding showing the upper subcoil only. The HF CICC is rectangular and the LF CICC is squared. The double layer DL1 is in the high field region at the innermost part of the subcoil, and DL7 at the outermost part of the subcoil.

Table I

EDIPO Selected PARAMETERS AND STEADy State REsults

\begin{tabular}{|c|c|c|c|c|c|c|c|c|c|}
\hline & & & $\begin{array}{l}\text { DL1 } \\
\text { DL8 }\end{array}$ & $\begin{array}{l}\text { DL2 } \\
\text { DL9 }\end{array}$ & $\begin{array}{r}\text { DL3 } \\
\text { DL10 }\end{array}$ & $\begin{array}{r}\text { DL4 } \\
\text { DL11 }\end{array}$ & $\begin{array}{r}\text { DL5 } \\
\text { DL12 }\end{array}$ & $\begin{array}{r}\text { DL6 } \\
\text { DL13 }\end{array}$ & $\begin{array}{r}\text { DL77 } \\
\text { DL14 }\end{array}$ \\
\hline Length & $L$ & $\mathrm{~m}$ & $\overline{70.5}$ & 80.2 & 129.4 & $\overline{134.3}$ & $\overline{c 139.3}$ & $1 \overline{144.2}$ & 139.5 \\
\hline Number of turns & $N$ & - & 16 & 18 & 28 & 28 & 28 & 28 & 26 \\
\hline $\mathrm{Nb}_{3} \mathrm{Sn}$ cross section & $A_{S C}$ & $\mathrm{~mm}^{2}$ & 37.1 & 24.7 & 15.5 & 15.5 & 12.4 & 12.4 & 12.4 \\
\hline Copper cross section (total) & $A_{C u}$ & $\mathrm{~mm}^{2}$ & 37.1 & 49.5 & 40.2 & 40.2 & 43.3 & 43.3 & 43.3 \\
\hline Helium cross section & $A_{H e}$ & $\mathrm{~mm}^{2}$ & 37.9 & 37.9 & 24.6 & 24.6 & 24.6 & 24.6 & 24.6 \\
\hline Void fraction & $v_{f}$ & - & 0.33 & 0.33 & 0.3 & 0.3 & 0.3 & 0.3 & 0.3 \\
\hline Hydraulic diameter & $D_{h}$ & $\mathrm{~mm}$ & 0.482 & 0.482 & 0.417 & 0.417 & 0.417 & 0.417 & 0.417 \\
\hline Wetted perimeter & $P_{w}$ & $\mathrm{~mm}$ & 0.315 & 0.315 & 0.236 & 0.236 & 0.236 & 0.236 & 0.236 \\
\hline Coupling loss constant in $\mathrm{x}$ & $n \tau_{x}$ & $\mathrm{~ms}$ & 39.7 & 39.7 & 23.2 & 23.2 & 23.2 & 23.2 & 23.2 \\
\hline Coupling loss constant in y & $n \tau_{y}$ & $\mathrm{~ms}$ & 10.3 & 10.3 & 23.2 & 23.2 & 23.2 & 23.2 & 23.2 \\
\hline \multirow[t]{2}{*}{ Helium velocity (average) } & $v_{H e}$ & $\mathrm{~m} / \mathrm{s}$ & 0.470 & 0.440 & 0.300 & 0.290 & 0.285 & 0.280 & 0.285 \\
\hline & & & 0.485 & 0.460 & 0.310 & 0.305 & 0.295 & 0.295 & 0.297 \\
\hline \multirow[t]{2}{*}{ Helium residence time } & $t_{\text {res }}$ & $\mathrm{s}$ & 150.0 & 182.3 & 431.3 & 463.1 & 488.8 & 514.6 & 489.5 \\
\hline & & & 145.5 & 174.3 & 417.4 & 440.3 & 472.2 & 4885 & 468.9 \\
\hline
\end{tabular}

2) Heat Inputs: The heat generated from different sources and deposited into the superconducting system must be removed in order to keep the conductor temperature low enough to maintain the superconducting state. Two heat sources are included in the EDIPO CICC model: (a) heat generated by AC losses and (b) heat generated by the joint electrical resistance.

The AC losses consist of two contributions, i.e., coupling losses $\left(P_{C}\right)$ and hysteresis loss $\left(P_{H}\right)[6]$ :

$$
\begin{aligned}
P_{C x} & =\left(n \tau_{x} / 4 \pi 10^{-7}\right)\left(\dot{B}_{x}\right)^{2}(1+r) A_{S C} \\
P_{C y} & =\left(n \tau_{y} / 4 \pi 10^{-7}\right)\left(\dot{B}_{y}\right)^{2}(1+r) A_{S C} \\
P_{H} & =(2 / 3 \pi) d_{f} J_{c} \dot{B} A_{S C}
\end{aligned}
$$

where $x$ and $y$ are the local coordinate system in the conductor cross section. The time derivative of the magnetic field (components in $x$ and $y$ and resultant) is calculated as

$$
\dot{B}=(B / I)(d I / d t)
$$

where the field/current transfer functions (components and resultant) are calculated along $X$ by the ANSYS code using a finite element model which does not include the saddle-shaped ends for simplicity.
The heat generated by two joints located at inlet and outlet of each double layer is given by

$$
P_{J}=\alpha_{J} I^{2}
$$

where $\alpha_{J}=4 \mathrm{n} \Omega / \mathrm{m}$ is the electrical dissipation factor and the length of each joint is $0.5 \mathrm{~m}$. The joints are modeled with cross section and friction factor of the corresponding conductor.

\section{B. Tools}

The CryoSoft suite of codes provides optimized, flexible and validated tools for the analysis of specific issues in superconducting magnet systems, e.g., THEA (Thermal, Hydraulic and Electric Analysis of Superconductor Cables) [7] and FLOWER (Hydraulic Network Simulation) [8]. The new SUPERMAGNET code is used as the manager application: it launches two or more of the above codes, schedules their communication and terminates execution as appropriate [9].

For the EDIPO analysis the cryogenic system is analyzed analyzed with FLOWER, and the DC winding with 14 THEA codes, i.e., one code per double layer. These 15 processes and the managing SUPERMAGNET process run simultaneously. The heat generated by the Joule-Thomson effect due to helium expansion is accounted for in the THEA code. Each double layer 


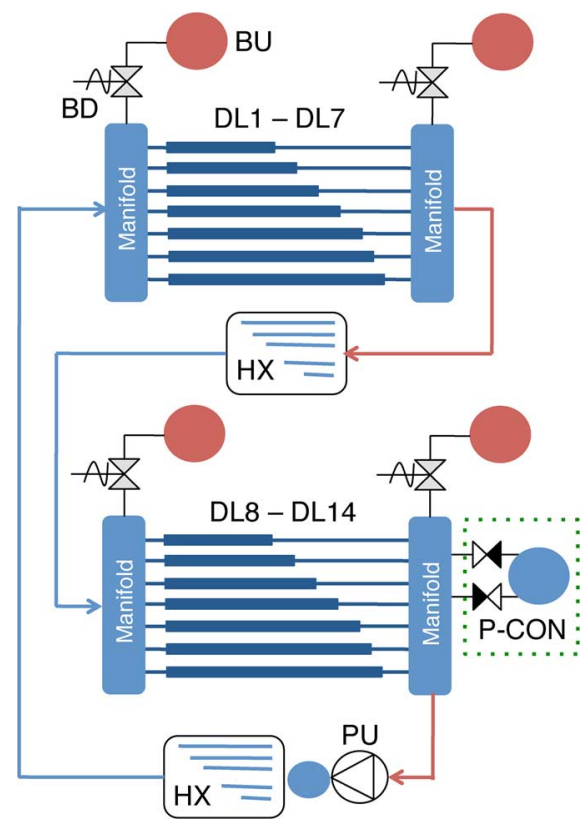

Fig. 2. Schematic representation of the EDIPO model. All 14 double layers are part of the winding model (DL1-DL7 in the upper coil, DL8-DL14 in the lower coil). Pump (PU), heat exchangers (HX), pressure control unit (P-CON), relief valves (BD), buffers (BU) and manifolds are part of the cryogenic model.

is modeled with 1000 elements. A CPU performance analysis with up to 200 simultaneous processes (the limit is given by the physical memory) confirms the robustness and scalability of this numerical tool.

\section{Steady State Analysis}

A steady state analysis $(I=0)$ is performed to check the validity of the model and to obtain the time at which the system has reached equilibrium following the initial numerical transient $\left(t_{\text {start }}\right)$, after which the current ramp is started.

The helium mass flow rate is $\approx 2.5 \mathrm{~g} / \mathrm{s}$ in the HF double layers (DL1, DL2, DL8 and DL9) and $\approx 1 \mathrm{~g} / \mathrm{s}$ in the LF double layers. Because of the different conductor lengths, the helium residence time (time to flow from inlet to outlet) varies between $\approx 150 \mathrm{~s}$ in the shortest double layers (DL1 and DL8) and $\approx 500 \mathrm{~s}$ in the longest one (DL6) (Table I).

During the residence time the helium temperature increases due to Joule-Thompson effect and joint electrical resistance. The difference between the temperature at the outlet and the inlet is $183 \mathrm{mK}$ in the upper subcoil and $88 \mathrm{mK}$ in the lower subcoil. These values differ because the helium pressure is 10 bar at the inlet of the upper subcoil and 7 bar at the inlet of the lower subcoil.

\section{CURREnt RAmp-Up ANALYSIS}

The sequence of the current model is: (a) no current until $t_{\text {start }}$ as in the steady state phase, (b) after $t_{\text {start }}$ a current ramp from 0 to $17 \mathrm{kA}$ with constant prescribed rates between 10 and $100 \mathrm{~A} / \mathrm{s}$, and (c) $I=17 \mathrm{kA}$ and constant until TH steady state conditions are reached. In all double layers $t_{\text {start }}=1000 \mathrm{~s}$.

The figure of merit for this analysis is the minimum value of the temperature margin in the DC winding $T_{M \min }$. In each simulation the results of $T$ and $T_{C S}$ are stored at all locations, at all times and in all double layers. Subsequently these data are used

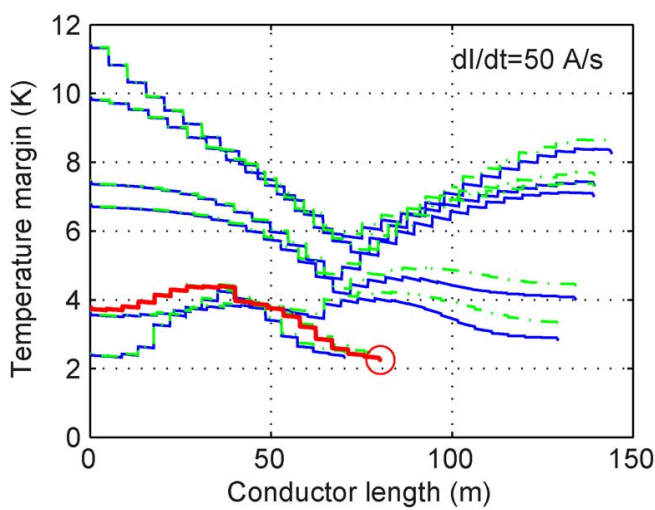

Fig. 3. Distribution of the temperature margin along the conductor length in each DL (solid lines: upper subcoil, dash-dotted line: lower subcoil) at the time at which $T_{M \min }$ occurs $(t=1340 \mathrm{~s})$. Reference case $(d I / d t=50 \mathrm{~A} / \mathrm{s})$. Value and location of $T_{M \min }$ are shown with a circle. The double layer DL2 is shown with a bold line.

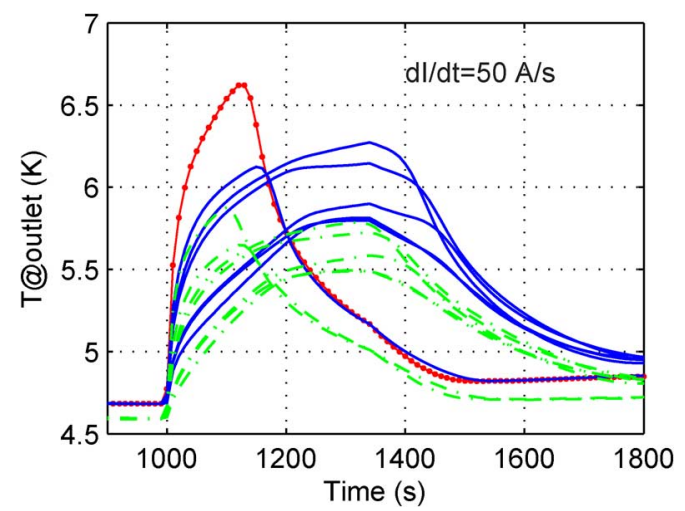

Fig. 4. Time history of conductor temperature at the outlet of each DL (solid lines: upper subcoil, DL1 with extra dots, dash-dotted lines: lower subcoil). Reference case $(d I / d t=50 \mathrm{~A} / \mathrm{s})$. The current ramp starts at $t_{\text {start }}=1000 \mathrm{~s}$ and ends at $t_{\text {end }}=1340 \mathrm{~s}$.

in an automated post processing procedure to find $T_{M \min }$, its location and time of occurence. The storage of this large amount of data is necessary since it is not known a priori where $T_{M \min }$ will occur. Incidentally, it is one of the major advantages of analyzing all double layers simultaneously, and to be able to post process the results of all double layers in one procedure.

\section{A. Reference Case}

The simulation with $d I / d t=50 \mathrm{~A} / \mathrm{s}$ is the "reference case" since this ramp rate is (close to) the highest value allowed by the EDIPO power converter.

The distribution of the temperature margin along the conductor length depends on the distribution of $T$ and $T_{C S}$ in $X$. Both distributions differ qualitatively in the double layers in HF and in LF (in HF, $T_{C S}$ is low, high, low and high at increasing $X$, in LF $T_{C S}$ is high, low, high), as shown in Fig. 3. In the reference case $T_{M \min }=2.253 \mathrm{~K}$ and occurs at the DL2 outlet $(X=80.2 \mathrm{~m})$ at $t=1340 \mathrm{~s}$, at the end of the current ramp $\left(t_{\text {end }}\right)$.

The conductor temperature at the outlet $T_{\text {out }}$ reaches the peak value at $t<t_{e n d}$ in $\mathrm{HF}$ and at $t=t_{\text {end }}$ in LF. This is due to the larger helium velocity in the former double layers (Table I). For example, the peak value $T_{\text {out }}=6.62 \mathrm{~K}$ in DL1 occurs after $130 \mathrm{~s}$ from the start of the current ramp (Fig. 4). 


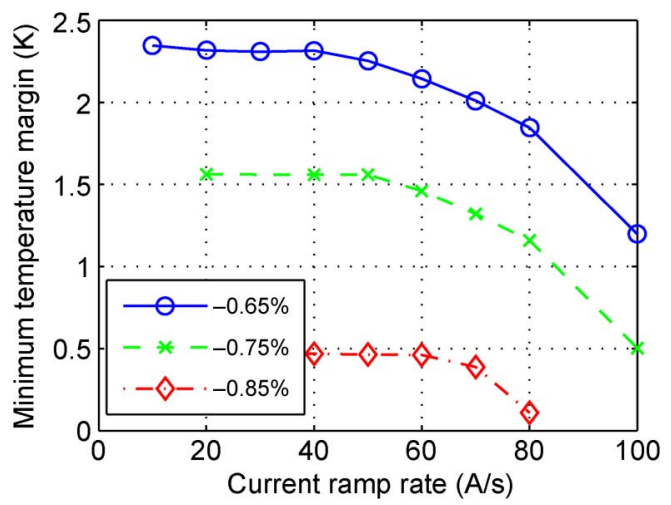

Fig. 5. Minimum temperature margin as a function of $d I / d t$ for 3 values of the longitudinal strain $\left(\epsilon=-0.65 \%\right.$ is the reference case). $T_{M \min }$ is approximately constant between 20 and $40 \mathrm{~A} / \mathrm{s}$ also for $\epsilon=-0.85 \%$ (the line below the legend is not visible).
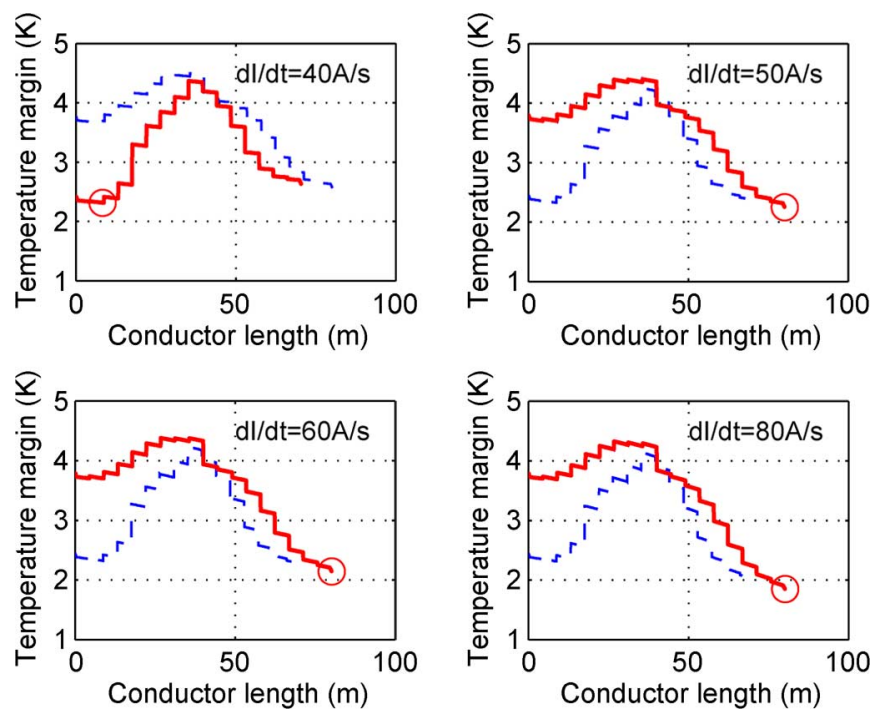

Fig. 6. Distribution of the temperature margin along the conductor length in DL1 and DL2 at the time at which $T_{M \min }$ occurs. The location of $T_{M \min }$ is shown with a circle and the corresponding DL with a solid line. Up to $40 \mathrm{~A} / \mathrm{s}$ $T_{M \text { min }}$ is near the DL1 inlet, whereas for higher $d I / d t$ it is at the DL2 outlet. At this location $T_{M \min }$ decreases for increasing $d I / d t$.

\section{B. Parametric Study}

The dependency of $T_{M \min }$ on $d I / d t$ is characterized by two distinct regimes: (a) at $d I / d t<40 \mathrm{~A} / \mathrm{s}, T_{M \min }$ does not depend on the current ramp rate, and (b) at $d I / d t>40 \mathrm{~A} / \mathrm{s} T_{M m i n}$ decays at increasing $d I / d t$, (Fig. 5). A reduction of the current ramp rate gives practically the same value of $T_{M \min }$ but at a different location in the DC winding. For example at $d I / d t=40 \mathrm{~A} / \mathrm{s}, T_{M \min }=2.32 \mathrm{~K}$ and occurs near the inlet of DL1 at $t=1740 \mathrm{~s}$, after the end of the current ramp $\left(t_{\text {end }}=1425 \mathrm{~s}\right.$ ) (Fig. 6). The EDIPO AC losses are dominated by the hysteresis losses which are independent of $d I / d t$. The reduction of $T_{M \min }$ in the regime (b) is caused by the reaction of the helium flow to the energy dissipated into the system: the energy is constant with $d I / d t$ whereas the power is not.

Degradation of the $\mathrm{Nb}_{3} \mathrm{Sn}$ superconductor is possible for a variety or reasons, e.g., coil manufacture, cycling during oper- ation, etc. We take this degradation into account by increasing the longitudinal strain $\epsilon$ from the measured value of $-0.65 \%$ (reference case) to a purely speculative value of $-0.85 \%$. At $d I / d t=50 \mathrm{~A} / \mathrm{s}, T_{M \min }$ is reduced from $2.25 \mathrm{~K}$ (reference case) to $0.46 \mathrm{~K}$ in the pessimistic case $(-0.85 \%)$. At any given $\epsilon$, the two regimes described above are still valid.

The sensitivity of $T_{M \min }$ to variation of the model parameters is very limited $(<1 \%)$ in case of the exponent $n$ (5 to 15$)$ and the joint electrical dissipation factor $\alpha_{J}(4$ to $8 \mathrm{n} \Omega / \mathrm{m})$. On the other hand, an increase of the helium inlet temperature from 4.5 $\mathrm{K}$ to $5 \mathrm{~K}$ corresponds to an almost identical reduction of $T_{M \min }$, i.e., $1.71 \mathrm{~K}$ compared to $2.25 \mathrm{~K}$ in DL1 at $X=8.4 \mathrm{~m}$.

\section{SUMMARY}

The current ramp-up analysis of the EDIPO DC magnet shows:

i) The simultaneous simulation of 14 double layers and the cryogenic system with the CryoSoft suite of codes, i.e., SUPERMAGNET to manage THEA and FLOWER, is feasible and provides a clear advantage for the assessment of the performance limits in the complete DC winding.

ii) When the EDIPO magnet is energized at the highest ramp rate allowed by the power converter $(50 \mathrm{~A} / \mathrm{s})$, the temperature margin is within acceptable values $(>2 \mathrm{~K})$.

iii) A reduction of the current ramp rate does not produce a relevant increase of the temperature margin. In case of major superconductor degradation this result may become a limitation, i.e., the temperature margin is too small.

iv) The complex and comprehensive EDIPO model was used in steady state and parametric analyses. Validation with experimental data is necessary and will be done when the EDIPO facility starts operation.

\section{ACKNOWLEDGMENT}

The authors are grateful tothank Maurizio Bagnasco (CRPP) for stimulating discussions on the cryogenic system and to Valeri Markushin (PSI) for IT support.

\section{REFERENCES}

[1] A. Portone et al., "Status of the EDIPO Project," IEEE Trans. Appl. Supercond., vol. 19, no. 3, pp. 1552-1556, 2009.

[2] A. Portone et al., Status of the EDIPO Project This conference.

[3] M. Calvi et al., "Preparatory work to host the EDIPO test facility at CRPP," IEEE Trans. Appl. Supercond., vol. 18, no. 2, pp. 204-207, 2008.

[4] L. T. Summers et al., "A model for the prediction of Nb3Sn critical current as a function of field, temperature, strain, and radiation damage," IEEE Trans. Magnetics, vol. 27, no. 2, pp. 2041-2044, 1991.

[5] C. Marinucci and G. Vécsey, "Heat front propagation and quench initiation analysis of the ITER toroidal field model coil," Cryogenics, vol. 40, pp. 501-510, 2000.

[6] C. Marinucci, L. Bottura, and M. Calvi, "A parametric AC loss model of the ITER coils for control optimization," Cryogenics, 10.1016/j. cryogenics.2009.06.003, accepted for publication.

[7] L. Bottura, C. Rosso, and M. Breschi, "A general model for thermal, hydraulic, and electric analysis of superconducting cables," Cryogenics, vol. 40, no. 8-10, pp. 617-626, 2000.

[8] L. Bottura and C. Rosso, "Flower, a model for the analysis of hydraulic networks and processes," Cryogenics, vol. 43 , no. 3-5, pp. 215-224, 2003.

[9] “SuperMagnet. User's Guide," ver. 1.0, CryoSoft User, 2007. 